\title{
CONJONCTURE
}

MARIE-EMMANUELLE POMMEROLLE

\section{LES VIOLENCES DANS L'EXTRÊME-NORD DU CAMEROUN : LE COMPLOT COMME OUTIL D'INTERPRÉTATION ET DE LUTTES POLITIQUES}

\begin{abstract}
Depuis 2013, la région de l'Extrême-Nord du Cameroun, frontalière du Nigeria et du Tchad, connaît des violences inédites. Bien que beaucoup d'entre elles aient été expressément revendiquées par Boko Haram, elles suscitent néanmoins de nombreuses interrogations. Des médias camerounais très populaires comme des intellectuels reconnus évoquent, souvent sous la forme interrogative ou par allusions, la complicité d'élites de la région ainsi que celle de «la France» dans cette "déstabilisation» du régime. La distance géographique, le manque d'informations et de connaissances sur les événements et leur contexte n'expliquent pas, à eux seuls, la prospérité de cette théorie du complot. L'article prend au sérieux ces rumeurs et ces théories complotistes en tentant de comprendre leur articulation aux imaginaires politiques disponibles, leurs canaux de diffusion et leur signification dans une période d'incertitude politique généralisée. Produit de luttes politiques, ces interprétations peuvent également être vues comme un moyen de faire l'économie d'une véritable réflexion sur les causes sociales et politiques de cette violence.
\end{abstract}

En décembre 2014, à Yaoundé, on entend deux versions complètement différentes concernant les violences qui ravagent l'Extrême-Nord du Cameroun depuis quelques mois. Située à près d'un millier de kilomètres du foyer des tensions, la capitale accueille alors deux récits qui ne s'affrontent ni ne se croisent. D'un côté, les quelques journalistes de l'unique bihebdomadaire disposant de véritables relais sur place exposent au reste du monde les attaques subies par les villages proches de la frontière nigériane et les forces de sécurité présentes dans la région¹. De l'autre, sur les ondes populaires, parmi les étudiants, dans les restaurants huppés comme dans les

1. Un seul journal, bihebdomadaire, L'CEil du Sahel, s'intéresse spécifiquement au Nord du pays et y possède un réseau de correspondants. Les autres journaux, notamment les quotidiens, disposent de très peu d'informations sur la région et n'en ont guère publié depuis le début des violences. 
Conjoncture

administrations, ces mêmes violences sont imputées, souvent à demi-mot, à quelques personnalités politiques de cette région et à «la France» qui les soutient. La force populaire et politique de ce second récit contraste avec la faible portée des faits recueillis minutieusement par L'CEil du Sahel qui comptabilise les villages brûlés, les bergers enlevés, le bétail volé. Alors qu'une mobilisation régionale «contre le terrorisme» s'engage depuis janvier 2015, le récit, jusque-là minoritaire, est maintenant validé par la parole officielle et repris par certains médias: désormais, il s'agit bien de combattre Boko Haram, et de soutenir les soldats camerounais engagés au front.

Cependant, les thèses complotistes n'ont pas disparu et la force de ce récit demeure une énigme politique à élucider. «Ce qui se dit» (la France soutenant Boko Haram, les élites du Nord certainement complices) est en soi important car ce discours politique et populaire participe concrètement de la (non-) gestion de ce conflit. Les conditions de production et de circulation de ce discours sont également à interroger pour en comprendre la teneur, l'ampleur et la spécificité. En effet, les accusations complotistes ont pu avoir cours au Nigeria également, mais le contexte historique et politique de leur production leur donne un sens différent: l'endogénéité du groupe islamiste n'y est pas remise en cause et les accusations de soutien à Boko Haram ou d'apathie face à cette insurrection sont liées au contexte électoral de ces derniers mois.

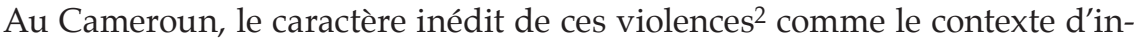
certitude politique ont ouvert un espace de parole extrêmement large, accueillant des tentatives d'explication et donc d'accusations multiples.

Nous commencerons par décrire l'évolution des violences dans la région à partir des sources disponibles, c'est-à-dire essentiellement des informations de L'CEil du Sahel désignant Boko Haram comme leur principal responsable. Nous relaterons ensuite les interprétations circulant à Yaoundé et tenterons d'en comprendre les ressorts, notamment le recours aux figures de l'ennemi de l'intérieur et de l'extérieur, une constante des dispositifs du pouvoir postcolonial au Cameroun. Alors que le contexte actuel est celui d'une succession incertaine et que les luttes s'aiguisent, politiques et médias, aux intérêts entremêlés, mobilisent sur des thématiques populaires. La distance géographique et sociale entre les populations de l'Extrême-Nord, la classe politique et les populations du Sud autorise cette déconnexion. Ceci permet également de faire l'économie d'une réflexion sur les origines de cette violence,

2. Sur l'histoire des activités criminelles et parfois violentes menées dans cette région transfrontalière, voir J. Roitman, Fiscal Disobedience: An Anthropology of Economic Regulation in Central Africa, Princeton, Princeton University Press, 2004; I. Saïbou, Les coupeurs de route. Histoire du banditisme rural et transfrontalier dans le bassin du Lac Tchad, Paris, Karthala, coll. «Terrains du siècle», 2010. 
qui est loin d'être seulement importée. Ainsi, les commentaires sur cette actualité sont d'autant plus important à analyser qu'ils révèlent la manière dont les marges de l'État, dont l'Extrême-Nord fait partie, sont considérées et gouvernées au Cameroun.

\section{ChRONOLOGIE des VIOLENCES DANS L'EXTRÊME-NORD DU CAMEROUN}

Boko Haram est un mouvement religieux sectaire implanté depuis 2003 dans l'État de Borno, frontalier de la région de l'Extrême-Nord du Cameroun ${ }^{3}$. La répression menée à partir de 2009 à son encontre par les forces nigérianes entraîne une radicalisation des violences au Nigeria ${ }^{4}$ et conduit le mouvement à utiliser progressivement le Cameroun comme espace d'approvisionnement. L'enlèvement d'une famille française en février 2013 attire l'attention internationale (mais également nationale) sur l'activité supposée de ce groupe armé au Cameroun. Les modalités de négociation et la stratégie adoptées par le gouvernement et les forces de sécurité camerounaises sont alors celles qui prévalent par la suite lorsque les activités de Boko Haram se diffusent sur le territoire: intermédiation des élites politiques locales (députés, chefs traditionnels) capables de naviguer entre Cameroun et Nigeria, mise en retrait de l'administration, mobilisation des forces spéciales - Brigade d'intervention rapide (BIR) notamment ${ }^{5}$. Cependant, malgré la présence de ces forces armées, les populations de l'Extrême-Nord ressentent de plus en plus la pénétration $\mathrm{du}$ mouvement, $\mathrm{du}$ fait de la répression conduite par les forces nigérianes d'abord, puis de l'arrivée massive de Nigérians menacés et installés dans des villages de la région et enfin, alors que les forces nigérianes se retirent à partir de juin 2014, par le mouvement lui-même.

Les premiers cadres de la secte se seraient réfugiés ponctuellement au Cameroun dès 2009, lors de la première grande offensive nigériane contre la secte, puis, de manière plus systématique à partir de mi-2013. Des attaques ciblées, parfois suivies de représailles de l'armée nigériane, se sont ensuite

\footnotetext{
3. Sur l'émergence du mouvement, ses origines religieuses, ses composantes sociologiques et son rapport à l'État, voir notamment A. Higazi, «Les origines et la transformation de l'insurrection de Boko Haram dans le Nord du Nigeria», Politique africaine, n 130, 2013, p. 137-164.

4. Sur l'escalade de la violence depuis 2009, voir A. Higazi, «Mobilisation into and against Boko Haram in North-East Nigeria», in K. Tall, M.-E. Pommerolle et M. Cahen (dir.), Collective Mobilisations in Africa. Enough is Enough!/Mobilisations collectives en Afrique.Ça suffit!, Leyde, Brill/Africa-Europe Group for Interdisciplinary Studies, 2015.

5. Le BIR (successeur du Bataillon léger d'intervention ou BLI créé en 1999) est le Bataillon d'intervention rapide, unité d'élite dirigée par un général israélien en retraite. Il a déjà œuvré dans l'Extrême-Nord pour lutter contre les coupeurs de route. Son effectif de 4000 hommes devrait passer à 6000 en 2015.
} 
Conjoncture

multipliées à partir du début de $2014^{6}$. Le front du conflit pénètre au Cameroun, notamment autour de la ville frontalière d'Amchidé, et y demeure jusqu'à aujourd'hui. Les attaques violentes contre des villages camerounais s'enchaînent et les premiers recrutements de jeunes Camerounais sont mis au jour début 2014. Les attaques s'accompagnent d'assassinats ciblés de chefs traditionnels7, d'enfants, ainsi que de messages de menaces à destination des populations. Face aux perturbations logistiques que connaît Boko Haram, du fait des saisies d'armes et de l'arrestation de certains de ses logisticiens au Cameroun, le groupe intensifie les attaques dans ce pays et met la région sous pression ${ }^{8}$. L'enlèvement de religieux européens et canadiens en avril 2014 peut être compris de la sorte, comme celui des travailleurs chinois le mois suivant. Dans les deux cas, les négociations semblent montrer que les enlèvements pourraient aussi être le fait de bandits locaux agissant de concert avec Boko Haram.

L'armée nigériane perdant du terrain entre juin et août 2014, les espaces frontaliers du Cameroun se retrouvent aux mains de la secte. Les Nigérians affluent alors en masse au Cameroun (en mai 2015, le Haut Comité des Nations unies pour les Réfugiés compte 74000 réfugiés nigérians au Cameroun) ${ }^{10}$. Les incursions visant à se ravitailler en armes, en nourriture et en bétail se multiplient et pénètrent, bien plus qu'avant, en territoire camerounais. En face, la stratégie militaire et politique camerounaise s'adapte: le gouverneur de la région, dépassé par les événements, est muté; une quatrième région militaire est créée dans le Nord au mois d'août. Les découvertes de caches d'armes et les arrestations se font plus nombreuses. Villageois et élites reconnaissent que le recrutement s'accélère et que des centaines de jeunes disparaissent ${ }^{11}$. Le nombre et le niveau de violence des attaques de Boko Haram s'intensifient, avec pour objectif probable de s'installer près du Lac Tchad, dans le Logone et Chari, une zone de confins, riche en ressources naturelles, en armes et en hommes disponibles (d'anciens rebelles tchadiens, notamment) ${ }^{12}$. Suite à la prise de la ville de Baga, au bord du Lac, les forces tchadiennes font route vers cette zone à partir de la mi-janvier 2015. Mais depuis septembre 2014, c'est tout l'Extrême-Nord qui connaît une recrudescence de la violence. Les incursions dans les villages sont quasi

6. L'CEil du Sahel, n 578, 20 février 2014.

7. L'CEil du Sahel, n 588, 27 mars 2014.

8. L'CEil du Sahel, n 591, 7 avril 2014.

9. L'CEil du Sahel, n 606, 2 juin 2014.

10. Agence des Nations unies pour les réfugiés (UNHCR), «Le chef du HCR António Guterres en visite auprès des réfugiés nigérians au Nord du Cameroun» [en ligne], 24 mars 2015, <www.unhcr. fr/5512873cc.html >, consulté le 15 mai 2015.

11. L'CEil du Sahel, $\mathrm{n}^{\circ} 607,5$ juin 2014.

12. L'CEil du Sahel, n 622, 29 juillet 2014. 
quotidiennes; les exécutions de chefs et parfois d'imams deviennent régulières; l'usage de roquettes et de mines ainsi que l'attaque directe des camps militaires se multiplient ${ }^{13}$.

Face à cette pression devenue difficilement soutenable à la fin de l'année 2014, le Cameroun s'est résolu à accepter le soutien militaire tchadien, puis la coopération internationale en janvier 2015. Jusque-là, les autorités camerounaises n'ont pas souhaité demander de soutien direct. Si les forces camerounaises n'ont pas subi de revers dramatiques, l'intensification des attaques et la débâcle nigériane semblent avoir convaincu le président Biya d'accepter du renfort pour l'armée camerounaise, qui travaille à la limite de ses capacités humaines, et plus particulièrement les forces spéciales. C'est ainsi que le gouvernement camerounais a autorisé l'armée tchadienne à pénétrer sur son territoire et que les réunions internationales se sont multipliées au Niger et au Cameroun, afin de mettre sur pied une force multinationale composée de plusieurs milliers d'hommes. Depuis février 2015, le rapport de forces militaire a donc changé, du fait de l'intervention tchadienne, du changement de stratégie nigériane (dans la perspective et à la suite des élections) et du soutien international. Si le niveau de coopération entre les armées de la région est considéré comme insuffisant ${ }^{14}$, il ne fait pas de doute que la multiplication des assauts dans la partie la plus septentrionale du Cameroun a permis une accalmie ${ }^{15}$. Les forces de Boko Haram n'ont cependant pas déserté le pays, se réfugiant dans la zone des monts Mandara ${ }^{16}$ où ils cherchent à s'approvisionner alors que, pour la secte, les espaces au Nigeria se réduisent. La viabilité de la coalition internationale étant loin d'être assurée, les violences et la lutte contre celles-ci au Cameroun restent d'actualité.

Cette mobilisation générale, nationale comme étrangère et notamment française, n'a cependant pas mis fin aux doutes exprimés sur les origines et les modalités de ces violences, qui prospèrent depuis plusieurs mois. Après la visite du ministre français des Affaires étrangères à Yaoundé le 21 février, qui a cru bon de préciser: «la France est l'amie du Cameroun», certains journaux s'interrogeaient sur les réels motifs de cette visite, et pointaient du doigt une nouvelle ingérence française ${ }^{17}$. D'autres épisodes médiatiques depuis lors ont relancé l'hypothèse d'un soutien français à la secte nigériane.

La vigueur et l'ampleur de ces interprétations commandent que l'on s'attache à en comprendre les ressorts: alors que les populations de l'Extrême-Nord sont confrontées à des attaques quotidiennes et à une situation économique

13. Voir le récit de trois mois d'attaques dans L'CEil du Sahel, n 673, 29 janvier 2015.

14. Voir par exemple L'CEil du Sahel, 15 mai 2015.

15. L'CEil du Sahel, 16 avril 2015.

16. L'CEil du Sahel, 12 mars 2015 et 11 mai 2015.

17. La Météo, 23 février 2015. 
Conjoncture

dramatique, comment expliquer que médias, intellectuels, rumeurs et élites politiques concentrent leurs commentaires (et pendant longtemps très peu d'actions) sur ce qui serait de simples manipulations politiques?

\section{LES LECTURES COMPLOTISTES DES VIOLENCES}

Entre juin et août 2014, à Yaoundé, se multiplient les prises de paroles politiques officialisant la lecture complotiste des violences dans l'ExtrêmeNord. Après le président de l'Assemblée nationale, c'est au tour du ministre délégué au Contrôle supérieur de l'État, Henri Eyebe Ayissi, de signer l'«Appel des élites de la Lékié» appelant à se battre contre Boko Haram et dénonçant les «complices de Boko Haram, principalement dans les parties septentrionales » et les «puissances extérieures »18. Dans la lignée des «manifestes » exprimant la loyauté des élites (et de leurs fiefs) au chef de l'État, celui-ci s'en distingue néanmoins par sa charge accusatrice à l'encontre de «certaines élites du Nord», et par la thèse affirmée d'un complot, alliant ces dernières et des «étrangers » visant le pouvoir en place. Alors que la thèse d'une rébellion nordiste, appuyée par des "forces étrangères", se diffuse déjà dans certains médias camerounais, et qu'un média français l'a reprise et confortée ${ }^{19}$, les prises de position politiques assoient officiellement cette lecture. Le discours du président de l'Assemblée comme le manifeste ne fonctionnent cependant que par allusions, sans entrer dans les détails de cette interprétation. Quel est le mode opératoire de ces acteurs et pourquoi agissent-ils de la sorte? Pourquoi des élites du Nord chercheraient-elles à déstabiliser leur région à travers une extrême violence? Pourquoi des étrangers, notamment français, auraient intérêt à semer le chaos? Avant de tenter d'interpréter la prospérité de ces récits, leurs fondements et leurs modes de circulation, reprenons les arguments qu'ils déploient ${ }^{20}$.

18. Mutations, 2 septembre 2014.

19. F. Pigeaud, «Cameroun: Paul Biya, après plus de trente ans de règne, est confronté à une rébellion» [en ligne], Médiapart, 26 août 2014, <//www.mediapart.fr/journal/international/260814/ cameroun-paul-biya-apres-plus-de-trente-ans-de-regne-est-confronte-une-rebellion $>$, consulté le 26 août 2014. La presse française est très commentée, si ce n'est lue, au Cameroun. En fonction des agendas et des stratégies locales, elle peut à la fois servir d'espace de validation de thèses circulant au Cameroun, comme ce fut le cas ici, et de repoussoir lorsqu'est mise en cause la légitimité d'un journal de l'ancienne métropole à parler d'affaires camerounaises. Ce fut le cas par exemple au mois de mars 2015 suite à la parution d'un article du Monde (Afrique) sur la santé du président et de son épouse. Voir «Le Monde tropicalisé utilise le mensonge comme ressource politique», $L a$ Météo, 6 avril 2015.

20. La section suivante décrivant les différents «faits» alimentant les théories du complot nordiste ou français reprend des éléments entendus lors de conversations à Yaoundé, des éléments 


\section{Les indices d'un complot}

D'abord, les attaques attribuées à Boko Haram au Cameroun, et tout particulièrement les enlèvements, ne ressembleraient pas à celles perpétrées au Nigeria. La secte y utilise des armes lourdes et y déploie des attaques de masse alors que les enlèvements ont été (au moins jusqu'à la fin 2014) le mode opératoire le plus courant au Nord du Cameroun, souvent sans revendication. Or, à quoi sert un enlèvement s'il n'est pas revendiqué? N'est-ce pas le signe que Boko Haram n'est pas impliqué dans ces méfaits? Cet argument est notamment utilisé au moment de l'attaque de Kolofata en juillet 2014, lorsque la maison du vice-Premier ministre Amadou Ali est attaquée, son frère tué et sa femme enlevée. Selon ces interprétations, cette attaque relèverait d'une manipulation purement politique, visant à détourner l'attention de cette personnalité soupçonnée d'être l'un des instigateurs de la violence. Par ailleurs, la logistique armée du groupe pose question et de nombreux indices pointent la France comme principale complice de ces violences: les armes récupérées sur les lieux du conflit (armes israéliennes ${ }^{21}$, char portant l'inscription «2DB $»^{22}$ ) pourraient provenir de forces françaises; l'atterrissage inopiné à Kano d'un avion russe transportant des hélicoptères français confirmerait cette implication 23 ; en décembre 2013, les arrêts des bataillons français se rendant en Centrafrique (arrêts près de Yaoundé, à Ngaoundéré), soi-disant non prévus, seraient en fait des moyens de positionner des troupes.

L'ensemble de ces «indices » et autres «faits troublants», disséminés dans les médias par certains personnages politiques et dans les conversations ordinaires dévoilerait la stratégie politique sous-tendant cette violence inédite. L'objectif serait de créer du désordre pour forcer le régime camerounais à ouvrir le dialogue politique avec «le Nord » et notamment avec l'un des hommes forts de la région, Marafa Hamidou Yaya, emprisonné depuis avril 2012 pour faits de corruption, qui compterait de nombreux soutiens à la fois parmi les «élites du Nord», mécontentes du sort fait à leur héraut, et en France. Ancien ministre de l'Administration territoriale, cette «élite du

diffusés lors d'émissions radiophoniques, ou par les médias écrits. Les sources ne sont donc pas toujours présentées de façon détaillée.

21. F. Pigeaud, «Cameroun: Paul Biya...», art. cité.

22. Cette inscription renverrait à la deuxième division blindée du général Leclerc. Ce fait troublant a été entendu lors de discussions diverses en décembre 2014.

23. D'autres explications d'inspiration complotiste ont pu être trouvées à cet incident: au Nigeria, on évoque l'hypothèse d'un soutien du Tchad à Boko Haram, ou encore la sécurisation des intérêts français au Nord-Est du Nigeria, menacés par Boko Haram: RFI, «Nigeria: l'Antonov rempli de matériel militaire français peut repartir» [en ligne], 8 décembre 2014, <www.rfi.fr/afrique/20141208nigeria-antonov-124-materiel-militaire-francais-peut-partir/>, consulté le 8 décembre 2014. 
Conjoncture

Nord» entretiendrait en effet un lien fort avec la France, dont il aurait été le protégé sous la présidence de Nicolas Sarkozy. La France soutiendrait donc cette rébellion pour contrôler la succession et préserver ses intérêts et ceux des entreprises françaises. En effet, le Cameroun est un pays stratégique dans une région en proie aux difficultés. Les intérêts français y sont bien implantés. Bolloré, qui détient la concession du port de Douala et de multiples autres entreprises agricoles et de transport serait, par exemple, un grand ami de Marafa. Ces positions doivent être maintenues mais aussi défendues face à une perte du monopole français sur les richesses camerounaises, notamment dans l'Extrême-Nord: des entreprises chinoises y sont par exemple en charge de l'exploration pétrolière. L'enlèvement des ouvriers chinois dans la région en mai 2014 aurait ainsi coïncidé avec l'annonce de la découverte de pétrole par les entreprises chinoises et pourrait, toujours selon cette interprétation complotiste, être attribué à des sous-traitants des intérêts français.

L'intervention française au Cameroun serait facilitée par la présence de l'ancienne puissance coloniale dans toute la région sahélienne. La proximité des foyers de violence avec le centre de commandement de l'opération Barkhane, à N'Djamena, ne serait en effet pas fortuite et le fait que le chef de cette opération, soit le même que celui qui commandait l'opération Licorne en Côte d'Ivoire, vient nourrir l'idée d'une stratégie identique à celle de l'insurrection ivoirienne, soutenue de bout en bout par la France afin d'écarter le président Gbagbo et de placer un proche de la France, Alassane Ouattara ${ }^{24}$.

\section{Les multiples figures des ennemis de l'intérieur et de l'extérieur}

Ce raisonnement par analogie permet d'ancrer l'interprétation complotiste des violences dans le précédent ivoirien qui fait particulièrement sens au Cameroun. La crise ivoirienne y a été suivie avec beaucoup d'attention, notamment au moment de l'intervention française en 2011. Celle-ci a fait l'objet d'intenses condamnations dans les sphères intellectuelles comme dans les conversations ordinaires ${ }^{25}$. Surtout, les interprétations de la crise ivoirienne sont fondées sur des clivages grossiers mais efficaces qui servent également de grille de lecture politique au Cameroun: le clivage Nord/Sud et le «nationalisme anti-français ». Le recours à ces antagonismes pour lire les violences actuelles s'insère dans une longue histoire de désignation d'ennemis de l'intérieur et de l'extérieur dans les périodes de crise et ce, depuis l'indépendance.

24. Le chef de l'opération Barkhane est le général Jean-Pierre Palasset qui a commandé l'opération Licorne, en Côte d'Ivoire, en 2010-2011 au moment de la chute de Laurent Gbagbo.

25. Voir, par exemple, les articles de la rubrique «Manières de voir» de la revue Germinal (Yaoundé). 
En 1960, le nouvel État fait face à une insurrection armée, notamment dans l'Ouest du pays. Prolongement du mouvement armé lancé par l'Union des populations du Cameroun (UPC) à partir de son interdiction en 1955, cette insurrection, ancrée dans les dynamiques internes aux sociétés dites des Grassfields, engendre une répression sévère de l'armée camerounaise soutenue par l'armée française ${ }^{26}$. En plus d'une coercition violente, l'arsenal juridique et la propagande politique construisent alors la figure du «terroriste » ou du «subversif » comme ennemi de la nouvelle nation. Jusqu'au début des années 1980 au moins, cette figure sert de justification à une politique autoritaire et est brandie à l'encontre de différents groupes sociaux jugés menaçants: «Bamilékés», «étudiants», «opposants» en général servent ainsi régulièrement de figure de l'ennemi de la nation et du jeune État en construction. Alors que Paul Biya, le successeur d'Ahidjo en 1982, promettait une «libéralisation communautaire», la désignation ethnique ou régionaliste des ennemis de l'intérieur prospère dès le début de son mandat, puis davantage encore lors du retour du multipartisme en 199027. Les «Nordistes», sur lesquels nous revenons ensuite, «les Bamilékés», les anglophones ${ }^{28}$, rassemblés parfois sous la catégorie «anglo-bamis» et tous les «opposants» en général sont montrés du doigt. Cette construction régionaliste des bons et des mauvais citoyens renvoie à «l'équilibre régional », un des outils de l'ordre politique au Cameroun. Institutionnalisé dans les concours de la fonction publique mais aussi dans les nominations politiques et administratives, cet équilibre qui aurait favorisé un ordre pacifique, solidifie les appartenances et sert également d'armes et de registres politiques. Ainsi, les «Nordistes», aujourd'hui accusés de déstabiliser le pouvoir au travers de leurs complicités avec Boko Haram, sont passés d'une catégorie à l'autre en fonction des configurations politiques.

Alors même que la consistance d'une identité nordiste ou sudiste paraît bien légère, et que les luttes de clan, notamment au sein du parti au pouvoir, sont à la fois plus localisées et transrégionales, le registre régionaliste est activé dans les moments d'incertitude ${ }^{29}$. Après avoir été favorisés lors de la présidence

26. Sur cette insurrection, voir D. Malaquais, Architecture, pouvoir et dissidence au Cameroun. Paris/ Yaoundé, Karthala/Presses de 1'UCAC, 2002; M. Terretta, Nation of Outlaws, State of Violence. Nationalism, Grassfields Tradition, and State Building in Cameroon, Athens, Ohio University Press, 2013. Sur la répression, voir T. Deltombe, M. Domergue et J. Tatsitsa, Kamerun! Une guerre cachée aux origines de la Françafrique, 1948-1971, Paris, La Découverte, coll. «Cahiers libres», 2011.

27. P. Geschiere, The Perils of Belonging. Autochthony, Citizenship and Exclusion in Africa and Europe, Chicago, Chicago University Press, 2009.

28. Ces accusations s'amplifient à partir du moment où des revendications autonomistes émergent, mais surtout du fait de la popularité du Front social démocrate créé en 1990 et dirigé par des anglophones.

29. Cette lecture régionaliste des luttes politiques fut également celle de certains acteurs et observateurs politiques lors de la bataille suivant la succession présidentielle entre Ahmadou 
Conjoncture

Ahidjo, les «Nordistes» ont rapidement été désignés comme responsables de la déstabilisation du régime lors de la bataille de succession de 1983-1984 ayant suivi la prise de pouvoir de Paul Biya. Suite à une tentative de coup d'État, plusieurs centaines de personnalités politiques mais surtout militaires originaires du Nord, comme l'ancien président, ont été exécutées ou apostasiées de la nation. Depuis, l'identification «nordiste» s'appuie sur cet épisode et sur d'autres vexations, pour rassembler autour d'une victimisation commune ${ }^{30}$. Plus récemment, les condamnations d'hommes politiques de premier plan, comme Marafa Hamidou Yaya ou encore Iya Mohammed ${ }^{31}$, sont utilisées pour souligner les abus dont «le Nord» serait victime. Ces stigmates sont aussitôt retournés pour revendiquer l'idée d'une prise de pouvoir nécessaire par ces mêmes ressortissants du Nord. De leur côté, certaines élites du Sud, craignant de perdre leur position privilégiée dans les arcanes du pouvoir (elles sont proportionnellement plus nombreuses à occuper des postes enviés ${ }^{32}$ ), orchestrent également la menace que représenterait le pouvoir des «Nordistes»: à partir de 2013, apparaît la rumeur de la constitution d'un «parti du Grand Nord» auquel appartiendraient secrètement des membres du Rassemblement démocratique du peuple camerounais (RDPC) ${ }^{33}$; en 2014, ces mêmes élites sont accusées d'être derrière les attaques de l'Extrême-Nord. La consolidation progressive de ce clivage, également fondée sur une méconnaissance et l'éloignement entre ces différentes régions du pays, favorise les interprétations complotistes actuelles mettant en cause la «loyauté» des élites du Nord. Le fait qu'elles soient également brandies par des élites du Nord, à l'encontre de leurs «compatriotes » et que la même mécanique accusatoire ait été déployée au sujet de certaines élites du Sud à l'approche des dernières élections présidentielles, montre néanmoins combien ces identifications sont souples, et liées à des conjonctures politiques labiles ${ }^{34}$.

Ahidjo et Paul Biya en 1983-1984: J.-F. Bayart, «La société politique camerounaise (1982-1986)», Politique africaine, $\mathrm{n}^{\circ} 22,1986$, p. 5-35.

30. Le débat récurrent autour du retour de la sépulture du premier président enterré à Dakar, car mort en exil, ravive régulièrement l'idée d'une victimisation du Nord.

31. Iya Mohammed est l'ancien directeur général de la Sodecoton et président de la Fédération camerounaise de football. Il a été accusé et emprisonné pour détournement de fonds en juin 2013. 32. Voir les tentatives d'objectivation de cette répartition régionale et départementale des postes politiques et administratifs par L'CEil du Sahel, n 537, 24 juin 2013 et n 589, 31 mars 2014 contenant chacun une édition spéciale intitulée «Le Gâteau national».

33. Entre 2007 et 2011, dans la perspective de l'élection présidentielle, un groupe de personnalités politiques, pour la plupart originaires de régions du Sud, ont été désignées comme appartenant à un mystérieux «G11 » soupçonné de vouloir prendre le pouvoir. Accusées de corruption, nombre d'entre elles ont été emprisonnées.

34. Les luttes entre élites du Nord sont considérables: c'est en effet le président de l'Assemblée nationale, Cavaye Yeguie qui a été l'un des premiers à désigner d'autres personnalités politiques de la région comme complices voire instigatrices des violences. L'CEil du Sahel, n 609, 12 juin 2014, $\mathrm{n}^{\circ} 618,14$ juillet 2014 et $\mathrm{n}^{\circ} 624,4$ août 2014. 
Au Cameroun, l'ennemi n'est pas qu'intérieur. Des ennemis extérieurs ont pu être désignés comme principal soutien d'un régime honni (la France) ou comme cherchant à déstabiliser le régime (les États-Unis au début des années 1990, la France plus récemment). L'accusation actuelle portée à la France, principal ennemi extérieur, révèle la force d'un «sentiment anti-français» fondé sur un récit nationaliste ancien et partagé. L'exutoire conjoncturel que représente la cible française s'appuie en effet sur l'histoire très riche d'un projet national considéré comme inachevé car entravé par la France. Il renvoie à la lutte de l'UPC, sévèrement réprimée par les forces coloniales, à la suppression de la mémoire de ces événements par les régimes successifs $^{35}$ et au rôle accordé à la France dans le maintien au pouvoir de régimes postcoloniaux illégitimes ${ }^{36}$. Cette forme de nationalisme, dirigé contre la France, s'est nourrie plus récemment d'un ensemble d'interventions françaises en Afrique, largement commentées dans les médias et les conversations ordinaires au Cameroun. L'intervention en Libye et l'assassinat de Mouammar Kadhafi tout comme le soutien français apporté à Alassane Ouattara contre Laurent Gbagbo y sont amplement condamnés. Ce nationalisme et ce souverainisme participent aussi d'un discours politique brandi par les élites au pouvoir. Au nom de la souveraineté camerounaise, il n'est pas rare en effet d'entendre certains ministres dénoncer vertement des initiatives ou des prises de position des ambassades occidentales présentes à Yaoundé37. L'accumulation de ces différentes strates d'anticolonialisme et d'anti-impérialisme et leurs usages divers se cristallisent dans cette accusation portée à la France d'être derrière les violences de l'Extrême-Nord. La multiplication des dénégations de la part de l'ambassade française au Cameroun et des personnalités politiques qui s'y rendent démontrent, s'il le fallait, l'ampleur de ce discours anti-français ${ }^{38}$. Les commentaires de la presse camerounaise

35. A. Mbembe, «L'État-historien», in R. Um Nyobé, Écrits sous maquis, Paris, L'Harmattan, 1989. 36. Voir par exemple les écrits de Mongo Béti, célèbre romancier et pourfendeur du néo-colonialisme français, notamment au Cameroun: Main basse sur le Cameroun. Autopsie d'une décolonisation, publié d'abord chez Maspero en 1972, interdit ensuite, puis re-publié en 1977 et à nouveau en 2010 aux éditions de la Découverte. L'écrivain Eyoh Meyonesse nous livre une prise de position plus actuelle: «Opinion: La désignation des dirigeants camerounais par la France: d'hier à aujourd'hui», Le Messager, 6 décembre 2014.

37. Ce fut le cas à l'encontre des diplomates qui s'interrogeaient sur la composition de l'institution «indépendante» chargée de l'organisation des élections. Voir notamment «Elecam: Le Minrex menace le corps diplomatique», Mutations, 20 février 2009.

38. Voir, entre autres, la lettre de l'ambassadrice de France au Cameroun publiée par Mutations, 31 décembre 2014; le discours de Laurent Fabius qualifiant l'implication française aux côtés de Boko Haram de «fiction» dans Le Jour, 23 février 2015, ou encore le communiqué de l'ambassadrice démentant les informations délivrées par le ministre tchadien de la Communication selon lesquelles $40 \%$ des armes récupérées sur les troupes de Boko Haram seraient d'origine française, Le Jour et Le Messager, 10 mars 2015. 
Conjoncture

sur ces efforts remarqués s'amusent de ces démonstrations d'amitié et, pour certains, valident à demi-mot les accusations portées ${ }^{39}$.

\section{Incertitude, luttes politiques et loyauté}

Les interprétations complotistes s'insèrent ainsi dans un registre historiquement disponible. Leur production et leur circulation sont également favorisées par les conditions politiques contemporaines et le contexte de luttes politiques et médiatiques, dans la perspective d'une succession attendue mais très incertaine. Âgé aujourd'hui de 82 ans, l'actuel président pourrait se représenter en 2018, passer la main auparavant, ou encore être empêché. La figure de Paul Biya étant intouchable, il est difficile de parler ouvertement de cette succession qui laisse chacun à ses spéculations. Élites comme militants du parti au pouvoir s'interrogent à voix basse sur l'avenir, évoquant le spectre de conflits internes au parti et d'une "Nuit des longs couteaux», lorsque l'heure de la succession aura sonnét0. La désignation d'élites politiques du Nord comme responsables des violences, tant par leurs homologues du Nord comme du Sud, résulte de ce contexte. Les préparatifs politiques, discrets, vont ainsi bon train et passent notamment par le soutien à de nombreux groupes de médias dont l'objectif est de mobiliser pour leur bienfaiteur.

Dans ces conditions, la théorie du complot nordiste et surtout français est politiquement rentable car extrêmement populaire. Elle est largement véhiculée par les médias, soutenus par des élites politiques, qui accueillent «intellectuels» et débats autour de cette thèse. La chaîne Afrique média qui se veut panafricaine, véhicule par exemple depuis plusieurs mois la thèse de l'implication française. Soutenue financièrement par des réseaux liés à la Guinée équatoriale, la chaîne est extrêmement suivie au Cameroun et les rumeurs courent sur la volonté politique française de la faire fermer ${ }^{41}$. D'autres médias comme ceux du groupe l'Anecdote diffusent ce type de discours ${ }^{42}$. Nombreuses sont également les figures liées au monde intellectuel local qui promeuvent les thèses rattachant cette nouvelle intervention aux nombreuses

39. «Pourquoi la France se sent coupable», Émergence, 12 mars 2015; «8 mars 2015. La France veut améliorer son image», La Nouvelle Expression, 8 mars 2015; «Extrême-Nord, à quoi joue la France?», Mutations, 26 mars 2015.

40. Expression entendue à plusieurs reprises lors d'entretiens avec des députés et militants du RDPC en décembre 2014.

41. R. Ndonkou, «Cameroun: la France accusée de chercher à tuer Afrique Media» [en ligne], Cameroon-Info.net, 25 novembre 2014,<www.cameroon-info.net/reactions/@,64405,7,camerounla-france-accusee-de-chercher-a-tuer-afrique-media.html $>$, consulté le 25 novembre 2015.

42. Informations collectées à Yaoundé, janvier 2015. 
ingérences passées de l'ancienne puissance coloniale ${ }^{43}$. Participant d'une sorte de convivialité entre "grands» et «petits», la thèse du complot consolide l'identification nationale et produit l'expression d'une loyauté et d'un unanimisme dans le soutien à un pouvoir menacé.

Alors que le président de la République a clairement désigné Boko Haram comme l'ennemi du Cameroun lors de son discours du 31 décembre et que Shekau, leader de cette secte, a revendiqué les attaques contre le pays, les interprétations complotistes, notamment celles dirigées contre la France, sont désormais concurrencées par d'autres interprétations et d'autres formes de démonstration de patriotisme et de loyauté au pouvoir. Certains médias, comme La Météo ont ainsi revu leur discours et se félicitent désormais de la coopération française dans la lutte contre les violences, alors qu'ils étaient les principaux canaux de diffusion du complot français ${ }^{44}$. La stratégie gouvernementale ayant évolué (engagement militaire, coopération internationale), les positionnements politiques et médiatiques se redessinent. Les expressions de soutien à l'armée camerounaise sont désormais les attributs nécessaires de l'élite politique. Les soldats morts au combat sont les nouveaux héros populaires, alors que les populations civiles, victimes de violences extrêmes depuis 2013, n'avaient pas suscité d'émotion particulière. Les manifestations de solidarité nationale se multiplient selon les formes habituelles de la mobilisation politique au Cameroun: organisation officielle de marches de soutien ou collecte de dons durant lesquelles les élites démontrent leur générosité ${ }^{4}$. Les expressions encadrées du patriotisme suscitent alors quelques débats politiques, qui n’ont cependant pas à voir avec les violences du Nord, mais suivent les logiques habituelles des échanges entre gouvernement et opposition ${ }^{46}$.

43. Les analyses critiques diffusées par Mongo Béti dans les années 1970 constituent le socle de ce discours qui s'est ensuite développé sous différentes formes. Elles sont relayées notamment au sein de l'association Survie. Les interprétations actuelles sur Boko Haram ne font cependant pas l'unanimité dans les milieux intellectuels camerounais, comme le montrent certains articles publiés dans le journal Germinal.

44. Voir par exemple «Performance en hausse-Christine Robichon», La Météo, 8 mars 2015 ou encore «Un procès inutile contre le France», La Météo, 12 mars 2015.

45. Une importante manifestation de solidarité envers les forces armées et les populations de l'Extrême-Nord a été organisée le 28 février dernier, d'abord par un groupe de journalistes originaires de la région, dont le directeur de publication de L'CEil du Sahel, puis a été intégrée à un dispositif officiel, plaçant cette marche dans la continuité des défilés organisés par le régime. Sur ces dispositifs de mobilisation et d'expression de la loyauté, voir N. Ngaméni et M.-E. Pommerolle, «Fabrics of Loyalty: The Politics of International Women Day's Wax Print Cloth in Cameroon», Africa, vol. 85, n 4, 2015 (à paraître); sur la mobilisation des élites, voir par exemple R. Orock, "Welcoming the "Fon of Fons" : Anglophone Elites and the Politics of Hosting Cameroon's Head of State», Africa, vol. 84, n² 2, 2014, p. 226-245.

46. Le choix de la date du 28 février pour la manifestation de solidarité avec l'Extrême-Nord est contesté par certaines personnalités politiques et militantes car elle renvoie à la répression des 
Répertoire d'imaginaires politiques disponibles, luttes intestines au sein du parti au pouvoir, expression d'une forme d'opposition ${ }^{47}$, moyen de détourner l'attention des échecs de l'État dans cette région, conviction parfois, concourent à faire de la théorie de l'insurrection nordiste soutenue par «les Français» l'un des discours les plus populaires ces derniers mois au Cameroun. Dans les moments de crise (crise de succession en 1983-1984, revendications démocratiques en 1990-1992, fin de règne depuis 2011), ces théories puisent dans le réservoir d'ennemis préexistant et permettent d'expliquer, d'anticiper et de réduire l'incertitude. Elles permettent aussi de faire l'économie de toute réflexion, et pendant de longs mois de toute action, sur ce qui se passe dans l'Extrême-Nord. Jusqu'en 2014, très peu d'informations ont été diffusées dans les journaux de la capitale sur les conditions de vie et de guerre dans cette région pourtant affectée par Boko Haram depuis 2013. Parler d'une rébellion fomentée par des ennemis déjà présents empêche que ne s'engage le moindre débat sur la pénétration relativement aisée de Boko Haram en territoire camerounais: intensification du recrutement de jeunes camerounais, absence de l'État et méfiance face à ses rares agents, illégitimité des élites sont, jusqu'à maintenant, passées sous silence dans les commentaires sur ces violences pourtant exceptionnelles sur ce territoire.

Marie-Emmanuelle Pommerolle Institut français de recherches en Afrique (IFRA)-Nairobi

\section{Abstract \\ Violence in the Far North Region of Cameroon: Conspiracy Theory and Political Struggles}

Since at least 2013, the region of the Far North of Cameroon, bordering Nigeria and Chad, is experiencing unprecedented violence. Although the attacks were for many expressly claimed by Boko Haram, whose base is in neighboring Nigeria, questions have been raised about the perpetrators of this violence. Popular Cameroonian media as well as intellectuals publicly mention, often as questions or allusions, the complicity of Northern elites and that of "France", seeking to "destabilize"

émeutes de février 2008 qui a fait une centaine de morts. Défiler un 28 février serait donc un moyen pour le pouvoir d'effacer les «héros» des uns pour les remplacer par de nouveaux héros de la nation.

47. Le discours de l'unique député du Mouvement pour la renaissance du Cameroun, parti d'opposition, à l'Assemblée nationale accusant directement la France d'être derrière Boko Haram, relevait certainement d'une volonté de se démarquer, mais aussi d'une forme de maladresse de la part d'un député novice, peu averti quant aux limites du dicible dans les enceintes officielles du pouvoir. Voir Le Messager, 2 décembre 2014. 
the regime. Geographical distance, lack of information and knowledge about the region cannot fully explain the prosperity of this conspiracy theory. This article seeks to unveil the origins of this theory by examining some patterns of the political imagination in Cameroon. It also examines the ways through which this theory is circulated, and the meaning of such discourses in a period of deep political uncertainty. Clearly the product of internal political struggles, these theories can also be seen as a way to avoid a true reflection on the social and political causes of violence in the region. 